\title{
Improving the Safety of Opioid Use for Acute Noncancer Pain in Hospitalized Adults: A Consensus Statement From the Society of Hospital Medicine
}

\author{
Shoshana J. Herzig, MD, MPH',2*, Hilary J. Mosher, MD³,4, Susan L. Calcaterra, MD, MPH,6,
} Anupam B. Jena, MD, PhD ${ }^{2,7}$, Teryl K. Nuckols, MD ${ }^{8}$

\begin{abstract}
${ }^{1}$ Department of Medicine, Beth Israel Deaconess Medical Center, Boston, Massachusetts; ${ }^{2}$ Harvard Medical School, Boston, Massachusetts; ${ }^{3}$ The Comprehensive Access and Delivery Research and Evaluation Center at the lowa City Veterans Affairs Healthcare System, lowa City, lowa; ${ }^{4}$ Department of Internal Medicine, Carver College of Medicine, University of lowa, lowa City, lowa; ${ }^{5}$ Department of Medicine, Denver Health Medical Center, Denver, Colorado; ${ }^{6}$ Department of Medicine, Division of General Internal Medicine, University of Colorado, Aurora, Colorado; ${ }^{7}$ Department of Medicine, Massachusetts General Hospital, Boston, Massachusetts; ${ }^{8}$ Division of General Internal Medicine, Cedars-Sinai Medical Center, Los Angeles, California.
\end{abstract}

Hospital-based clinicians frequently treat acute, noncancer pain. Although opioids may be beneficial in this setting, the benefits must be balanced with the risks of adverse events, including inadvertent overdose and prolonged opioid use, physical dependence, or development of opioid use disorder. In an era of epidemic opioid use and related harms, the Society of Hospital Medicine (SHM) convened a working group to develop a consensus statement on opioid use for adults hospitalized with acute, noncancer pain, outside of the palliative, end-of-life, and intensive care settings. The guidance is intended for clinicians practicing medicine in the inpatient setting (eg, hospitalists, primary care physicians, family physicians, nurse practitioners, and physician assistants). To develop the Consensus Statement, the working group conducted a systematic review of relevant guidelines, composed a draft
Statement based on extracted recommendations, and obtained feedback from external experts in hospital-based opioid prescribing, SHM members, the SHM PatientFamily Advisory Council, other professional societies, and peer-reviewers. The iterative development process resulted in a final Consensus Statement consisting of 16 recommendations covering 1) whether to use opioids in the hospital, 2) how to improve the safety of opioid use during hospitalization, and 3) how to improve the safety of opioid prescribing at hospital discharge. As most guideline recommendations from which the Consensus Statement was derived were based on expert opinion alone, the working group identified key issues for future research to support evidence-based practice. Journal of Hospital Medicine 2018;13:263-271. (C) 2018 Society of Hospital Medicine ince the initial reports of an emerging opioid epidemic in the early 2000s, intense focus on improving opioid prescribing in outpatient settings has culminated in new guidelines for chronic pain. ${ }^{1,2}$ Although opioid stewardship in the setting of chronic pain is of paramount importance in curbing the ongoing epidemic, longterm prescription opioid use often begins with treatment of acute pain. ${ }^{1}$ In addition to differences in recommended management strategies for acute and chronic pain, there are unique aspects and challenges to pain management in the acute-care setting.

Opioids are commonly used for the treatment of acute pain in hospitalized patients, often at high doses. ${ }^{3}$ Recent reports highlight that hospital use of opioids impacts downstream

*Address for correspondence: Shoshana J. Herzig, MD, MPH, Beth Israel Deaconess Medical Center, 330 Brookline Ave, CO-1309, Boston, MA 02215; Telephone: (617) 754-1413; Fax: (617) 754-1440

Received: December 28, 2017; Revised: February 23, 2018;

Accepted: March 5, 2018

2018 Society of Hospital Medicine DOI 10.12788/jhm.2980 use..$^{4.6}$ Additionally, opioid prescribing practices vary between hospital-based providers and hospitals,, 3 highlighting the need for prescribing standards and guidance. To our knowledge, there are no existing guidelines for improving the safety of opioid use in hospitalized patients outside of the intensive care or immediate perioperative settings.

The Society of Hospital Medicine (SHM) convened a working group to systematically review existing guidelines and develop a consensus statement to assist clinicians in safe opioid use for acute, noncancer pain in hospitalized adults.

\section{CONSENSUS STATEMENT PURPOSE AND SCOPE}

The purpose of this Consensus Statement is to present clinical recommendations on the safe use of opioids for the treatment of acute, noncancer pain in hospitalized adults. The guidance is intended for clinicians practicing medicine in the inpatient setting (eg, hospitalists, primary care physicians, family physicians, nurse practitioners, and physician assistants) and is intended to apply to hospitalized adults with acute, noncancer pain (ie, pain that typically lasts $<3$ months or during the period of normal tissue healing) outside of the palliative, end-of-life, and intensive care settings. 
TABLE 1. Topics for which Recommendations Were Extracted From Existing Guidelines

1. Deciding when to use opioids, nonopioid medications, and non-medication based pain management modalities

2. Best practices in screening/monitoring/education prior to prescribing an opioid and/or during treatment

3. Opioid selection considerations, including selection of dose, duration, and route of administration

4. Strategies to minimize the risk of opioid-related adverse events

5. Safe practices on discharge

\section{CONSENSUS STATEMENT DEVELOPMENT}

Our working group included experts in opioid use in the hospital setting, defined by 1) engagement in the clinical practice of hospital medicine and 2) involvement in clinical research related to usage patterns and clinical outcomes of opioid use in hospitalized patients (see Appendix Table 1). The SHM provided administrative assistance with the project and funded the in-person working group meeting, but it had no role in formulating the recommendations. The SHM Board of Directors provided approval of the Consensus Statement without modification.

An overview of the sequential steps in the Consensus Statement development process is described below; details of the methods and results can be found in the Appendix (eMethods).

\section{Performing the Systematic Review}

The methods and the results of the systematic review of existing guidelines on the management of acute pain from which the Consensus Statement is derived are described in a companion article. We extracted recommendations from each guideline related to the topics in Table 1 and used these recommendations to inform the Consensus Statement.

\section{Drafting the Consensus Statement}

After performing the systematic review, the working group drafted and iteratively revised a set of recommendations using a variation of the Delphi Method ${ }^{8}$ to identify consensus among group members.

\section{External Review}

Following agreement on a draft set of recommendations, we obtained feedback from external groups, including 1) individuals involved in the SHM's Reducing Adverse Drug Events Related to Opioids (RADEO) initiative, including those involved in the development of the implementation guide and site leads for the Mentored Implementation program, 2) SHM members, SHM Patient-Family Advisory Council (PFAC) members, and leaders of other relevant professional societies, and 3) peer-reviewers at the Journal of Hospital Medicine.

\section{RESULTS}

The process described above resulted in 16 recommendations (Table 2). These recommendations are intended only as guides and may not be applicable to all patients and clinical situations, even within our stated scope. Clinicians should use their judgment regarding whether and how to apply these recommendations to individual patients. Because the state of knowledge is constantly evolving, this Consensus Statement should be considered automatically withdrawn 5 years after publication, or once an update has been issued.

\section{Deciding Whether to Use Opioids During Hospitalization}

1. SHM recommends that clinicians limit the use of opioids to patients with 1) severe pain or 2) moderate pain that has not responded to nonopioid therapy, or where nonopioid therapy is contraindicated or anticipated to be ineffective.

Opioids are associated with several well-recognized risks ranging from mild to severe, including nausea, constipation, urinary retention, falls, delirium, sedation, physical dependence, addiction, respiratory depression, and death. Given these risks, the risk-to-benefit ratio is generally not favorable at lower levels of pain severity. Furthermore, for most painful conditions, including those causing severe pain, nonopioid analgesics, including acetaminophen and nonsteroidal anti-inflammatory drugs (NSAIDs), have been demonstrated to be equally or more effective with less risk of harm than opioids. ${ }^{9-13}$ Clinicians should consider drug-drug and drug-disease associations when deciding between these different therapies and make a determination in each patient regarding whether the benefits outweigh the risks. Often, drug-disease interactions do not represent absolute contraindications, and risks can be mitigated by adhering to dosage limits and, with respect to NSAIDs, 1) monitoring renal function, 2) monitoring volume status in patients with congestive heart failure, and 3) considering a selective cyclooxygenase-2 (COX-2) inhibitor rather than a nonselective NSAID or pairing the NSAID with an acid-suppressive medication in patients with a history of peptic ulcer disease or at elevated risk for gastroduodenal disease. For these reasons, a trial of nonopioid therapy (including pharmacologic and nonpharmacologic modalities) should always be considered before using opioids for pain of any severity. This does not imply that a trial of nonopioid therapy must be performed in all patients, but rather, that the likelihood of benefit and associated risks of opioid and nonopioid therapy should be considered for all patients in determining the best initial management strategy.

2. SHM recommends that clinicians use extra caution when administering opioids to patients with risk factors for opioid-related adverse events.

Several factors have been consistently demonstrated to increase the risk of opioid-related adverse events-most impor- 
TABLE 2. Society of Hospital Medicine Recommendations on Improving the Safety of Opioid Use for Acute Noncancer Pain in Hospitalized Adults Outside of Intensive Care, Palliative Care, and End-of-Life Care

Deciding Whether to Use Opioids During Hospitalization:

Limit the use of opioids to patients with 1) severe pain or 2) moderate pain that has not responded to nonopioid therapy, or where nonopioid therapy is contraindicated or anticipated to be ineffective.

Use extra caution when administering opioids to patients with risk factors for opioid-related adverse events.

Review the information contained in the prescription drug monitoring program (PDMP) database to inform decision-making around opioid therapy.

Educate patients and families or caregivers about potential risks and side effects of opioid therapy as well as alternative pharmacologic and nonpharmacologic therapies for managing pain.

Once a Decision Has Been Made to Use Opioids During Hospitalization:

Use the lowest effective opioid dose for the shortest duration possible.

Use immediate-release opioid formulations and avoid initiation of long-acting or extended-release formulations (including transdermal fentanyl) for treatment of acute pain

Use the oral route of administration whenever possible. Intravenous opioids should be reserved for patients who cannot take food or medications by mouth, patients suspected of gastrointestinal malabsorption, or when immediate pain control and/or rapid dose titration is necessary.

Use an opioid equivalency table or calculator to understand the relative potency of different opioids 1) when initiating opioid therapy, 2) when changing from one route of administration to another, and 3 ) when changing from one opioid to another. When changing from one opioid to another, clinicians should generally reduce the dose of the new opioid by at least $25 \%$ - $50 \%$ of the calculated equianalgesic dose to account for interindividual variability in the response to opioids as well as possible incomplete cross-tolerance.

Pair opioids with scheduled nonopioid analgesic medications, unless contraindicated, and always consider pairing with nonpharmacologic pain management strategies (ie, multimodal analgesia).

Unless contraindicated, order a bowel regimen to prevent opioid-induced constipation in patients receiving opioids.

Limit co-administration of opioids with other central nervous system depressant medications to the extent possible.

Work with patients and families or caregivers to establish realistic goals and expectations of opioid therapy and the expected course of recovery.

Monitor the response to opioid therapy, including assessment for functional improvement and development of adverse effects.

\section{Prescribing at the Time of Hospital Discharge:}

Ask patients about any existing opioid supply at home and account for any such supply when issuing an opioid prescription on discharge.

Prescribe the minimum quantity of opioids anticipated to be necessary based on the expected course and duration of pain that is severe enough to require opioid therapy after hospital discharge.

Ensure that patients and families or caregivers receive information regarding how to minimize the risks of opioid therapy for themselves, their families, and their communities. This includes but is not limited to: 1) how to take their opioids correctly (the planned medications, doses, schedule); 2) that they should take the minimum quantity necessary to achieve tolerable levels of pain and meaningful functional improvement, reducing the dose and/or frequency as pain and function improve; 3) how to safeguard their supply and dispose of any unused supply; 4) that they should avoid agents that may potentiate the sedative effect of opioids, including sleeping medication and alcohol; 5) that they should avoid driving or operating heavy machinery while taking opioids; and 6) that they should seek help if they begin to experience any potential adverse effects, with inclusion of information on early warning signs.

tantly, respiratory depression and overdose-in varied patient populations and settings, including age 65 years and older, ${ }^{1,14-17}$ renal insufficiency, ${ }^{1,14,18}$ hepatic insufficiency, ${ }^{1,14}$ chronic respiratory failure (including chronic obstructive pulmonary disease, sleep apnea, etc.), and receipt of other central nervous system (CNS) depressant medications (including, but not limited to, benzodiazepines). ${ }^{1,18-20}$ History of any substance use disorder and psychiatric disorders have been associated with an increased risk for the development of opioid use disorder. ${ }^{21-24}$ These factors should be weighed against the benefits when deciding on opioid appropriateness in a given patient. However, identification of these risks should not preclude opioids as part of pain management. When a decision is made to use opioids in patients with these risk factors, clinicians should 1) use a reduced starting dose (generally, at least a $50 \%$ reduction in the usual starting dose) and 2) consider closer monitoring for adverse effects (eg, more frequent nursing assessments, capnography, or more frequent outpatient visits).

\section{SHM recommends that clinicians review the informa- tion contained in the prescription drug monitoring program (PDMP) database to inform decision-making around opioid therapy.}

Although data on the impact of use of the state PDMP database on prescribing practices or patient outcomes are limited, PDMP use has been advocated by multiple guidelines on acute pain management. ${ }^{25-27}$ The PDMP provides information that can be useful in several ways, including 1) confirmation of prior opioid exposure and dosage, which should be used to guide appropriate dosage selection in the hospital, 2) identification of existing controlled substance prescriptions, which should be considered in prescribing decisions in the hospital and on discharge, and 3) identification of signs of aberrant behavior. For example, the identification of controlled substance prescriptions written by multiple different clinicians can facilitate early identification of potential diversion or evolving or existing opioid use disorder 
and the opportunity for intervention, ${ }^{28}$ which may include referral to support services, initiation of medication-assisted treatment, and/or pain specialist consultation when available. Concerns regarding evolving or existing opioid use disorder should prompt further discussion between the clinician and the patient, both to clarify their understanding of their recent prescription history and to discuss concerns for patient safety related to the increased risk of opioid-related adverse effects (including respiratory depression and overdose) among patients with controlled substance prescriptions written by multiple providers. Although such concerns should not automatically preclude the use of opioids for acute pain in the hospital setting, they should be considered in the assessment of whether the benefits of opioid therapy outweigh the risks for a given patient.

4. SHM recommends that clinicians educate patients and families or caregivers about the potential risks and side effects of opioid therapy as well as alternative pharmacologic and nonpharmacologic therapies for managing pain.

Patients are often unaware of the risks of opioid therapy (see Consensus Statement 1 for key risks), ${ }_{1}^{29}$ or that there are often equally effective alternative therapies. As with any therapy associated with substantial risk, clinicians should discuss these risks with patients and/or caregivers at the outset of therapy, as well as the potential benefits of nonopioid pharmacologic and nonpharmacologic therapies for managing pain. Patients should be informed that they may request nonopioid therapy in lieu of opioids, even for severe pain.

\section{Once a Decision Has Been Made to Use Opioids During Hospitalization}

5. SHM recommends that clinicians use the lowest effective opioid dose for the shortest duration possible.

Higher opioid doses are associated with an increased incidence of opioid-related adverse events, particularly overdose, in studies of both inpatient and outpatient populations. 17, 19,30,31 Studies in the inpatient and outpatient settings consistently demonstrate that risk increases with dosage. ${ }^{19,30,31}$ Clinicians should reduce the usual starting dose by at least 50\% among patients with conditions that increase susceptibility to opioid-related adverse events (see Consensus Statement 2). The ongoing need for opioids should be re-assessed regularly-at least daily-during the hospitalization, with attempts at tapering as healing occurs and/or pain and function improve.

6. SHM recommends that clinicians use immediate-release opioid formulations and avoid initiation of long-acting or extended-release formulations (including transdermal fentanyl) for treatment of acute pain.

Studies in outpatient settings demonstrate that the use of long-acting opioids is associated with greater risk for over- dose-especially in opioid-naïve patients-and long-term use. ${ }^{32,33}$ Further, hospitalized patients frequently have fluctuating renal function and rapidly changing pain levels. We therefore recommend that initiation of long-acting opioids be avoided for the treatment of acute, noncancer pain in hospitalized medical patients. It is important to note that although we recommend avoiding initiation of long-acting opioids for the treatment of acute, noncancer pain, there are circumstances outside of the scope of this Consensus Statement for which initiation of long-acting opioids may be indicated, including the treatment of opioid withdrawal. We also do not recommend discontinuation of long-acting or extended-release opioids in patients who are taking these medications for chronic pain at the time of hospital admission (unless there are concerns regarding adverse effects or drug-disease interactions).

\section{SHM recommends that clinicians use the oral route of} administration whenever possible. Intravenous opioids should be reserved for patients who cannot take food or medications by mouth, patients suspected of gastrointestinal malabsorption, or when immediate pain control and/or rapid dose titration is necessary.

Intravenous opioid administration is associated with an increased risk of side effects, adverse events, and medication errors. $^{34-36}$ Additionally, studies demonstrate that in general, the addiction potential of medications is greater the more rapid the onset of action (the onset of action is 5-10 min for intravenous and 15-30 minutes for oral administration). ${ }^{37,38}$ Furthermore, the duration of action is greater for oral compared to that of intravenous administration, potentially allowing for more consistent pain relief and less frequent administrations. As such, intravenous administration should be reserved for situations when oral administration is not possible or likely to be ineffective, or when immediate pain control and/or rapid titration is necessary.

8. SHM recommends that clinicians use an opioid equivalency table or calculator to understand the relative potency of different opioids 1) when initiating opioid therapy, 2) when changing from one route of administration to another, and 3) when changing from one opioid to another. When changing from one opioid to another, clinicians should generally reduce the dose of the new opioid by at least $25 \%-50 \%$ of the calculated equianalgesic dose to account for interindividual variability in the response to opioids as well as possible incomplete cross-tolerance.

Most errors causing preventable adverse drug events in hospitals occur at the ordering stage. ${ }^{39,40}$ Clinicians are often unaware of the potency of different types of opioids relative to each other or to morphine (ie, morphine equivalent dose), which can lead to inadvertent overdose when initiating therapy with nonmorphine opioids and when converting from one opioid to another. To facilitate safe opioid use, we recommend that clinicians use 
one of several available opioid equivalency tables or calculators to better understand the relative potencies of opioids and to inform both starting dose calculations and conversions between opioids and routes of administration. When converting from one opioid to another, we caution clinicians to reduce the dose of the new opioid by at least $25 \%-50 \%$ of the calculated equianalgesic dose to account for interindividual variability in the response to opioids and the potential for incomplete cross-tolerance, wherein tolerance to a currently administered opioid does not extend completely to other opioids. Clinicians should use extreme caution when performing conversions to and from methadone and consider consultation with a hospital pharmacist or a pain management specialist, when available, to assist with conversion decisions and calculations.

\section{SHM recommends that clinicians pair opioids with scheduled nonopioid analgesic medications, unless contraindicated, and always consider pairing with nonpharmacologic pain management strategies (ie, multimodal analgesia).}

Concurrent receipt of opioids and nonopioid analgesic medications (including acetaminophen, NSAIDs, and gabapentin or pregabalin, depending on the underlying pathophysiology of the pain) has been demonstrated to reduce total opioid requirements and improve pain management. ${ }^{41,42}$ Clinicians should be familiar with contraindications and maximum dosage recommendations for each of these adjunctive nonopioid medications. We recommend separate orders for each, rather than using drug formulations that combine opioids and nonopioid analgesics in the same pill, due to the risk of inadvertently exceeding the maximum recommended doses of the nonopioid analgesic (particularly acetaminophen) with combination products. We recommend that nonopioid analgesics be ordered at a scheduled frequency, rather than as needed, to facilitate consistent administration that is not dependent on opioid administration. Topical agents, including lidocaine and capsaicin, should also be considered. Nonpharmacologic pain management strategies can include procedure-based (eg, regional and local anesthesia) and nonprocedure-based therapies depending on the underlying condition and institutional availability. Although few studies have assessed the benefit of nonpharmacologic, nonprocedure-based therapies for the treatment of acute pain in hospitalized patients, the lack of harm associated with their use argues for their adoption. Simple nonpharmacologic therapies that can usually be provided to patients in any hospital setting include music therapy, cold or hot packs, chaplain or social work visits (possibly including mindfulness training), ${ }^{43}$ and physical therapy, among others.

\section{SHM recommends that, unless contraindicated, clinicians order a bowel regimen to prevent opioid-in- duced constipation in patients receiving opioids.}

Constipation is a common adverse effect of opioid therapy and results from the activation of mu opioid receptors in the colon, resulting in decreased peristalsis. Hospitalized patients are already prone to constipation due to their often-limited physical mobility. To mitigate this complication, we recommend the administration of a bowel regimen to all hospitalized medical patients receiving opioid therapy, provided the patient is not having diarrhea. Given the mechanism of opioid-induced constipation, stimulant laxatives (eg, senna, bisacodyl) have been recommended for this purpose. ${ }^{44}$ Osmotic laxatives (eg, polyethylene glycol, lactulose) have demonstrated efficacy for the treatment of constipation more generally (ie, not necessarily opioid-induced constipation). Stool softeners, although frequently used in the inpatient setting, are not recommended due to limited and conflicting evidence for efficacy in prevention or treatment of constipation. ${ }^{45}$ Bowel movements should be tracked during hospitalization, and the bowel regimen modified accordingly.

\section{SHM recommends that clinicians limit co-adminis- tration of opioids with other central nervous system depressant medications to the extent possible.}

This combination has been demonstrated to increase the risk of opioid-related adverse events in multiple settings of care, including during hospitalization. 1,18,19 Although benzodiazepines have received the most attention in this respect, other medications with CNS depressant properties may also increase the risk, including, but not limited to, nonbenzodiazepine sedative-hypnotics (eg, zolpidem, zaleplon, zopiclone), muscle relaxants, sedating antidepressants, antipsychotics, and antihistamines. ${ }^{18,19,46}$ For some patients, the combination will be unavoidable, and we do not suggest routine discontinuation of longstanding medications that preexisted hospitalization, given the risks of withdrawal and/or worsening of the underlying condition for which these medications are prescribed. Rather, clinicians should carefully consider the necessity of each medication class with input from the patient's outpatient providers, taper the frequency and/or the dose of CNS depressants when appropriate and feasible, and avoid new coprescriptions to the extent possible, both during hospitalization and on hospital discharge.

\section{SHM recommends that clinicians work with patients and families or caregivers to establish realistic goals and expectations of opioid therapy and the expected course of recovery.}

Discussing expectations at the start of therapy is important to facilitate a clear understanding of how meaningful improvement will be defined and measured during the hospitalization and how long the patient is anticipated to require opioid therapy. Meaningful improvement should be defined to include improvement in both pain and function. Clinicians should discuss with patients 1) that the goal of opioid therapy is tolerability of pain such that meaningful improvement in function can be achieved and 2) that a decrease in pain intensity in the absence of improved function is not 
considered meaningful improvement in most situations and should prompt reevaluation of the appropriateness of continued opioid therapy as well as close follow-up with a clinician following hospital discharge. Discussions regarding the expected course of recovery should include that acute pain is expected to resolve as the underlying medical condition improves and that although pain may persist beyond the hospitalization, pain that is severe enough to require opioids will often be resolved or almost resolved by the time of hospital discharge.

13. SHM recommends that clinicians monitor the response to opioid therapy, including assessment for functional improvement and development of adverse effects.

Pain severity and function should be assessed at least daily, and improvement in reported pain severity without improvement in function over several days should, in most circumstances, prompt reconsideration of ongoing opioid therapy and reconsideration of the underlying etiology of pain. Although hospital-specific functional measures in the setting of acute pain have not yet been validated, we suggest that such measures and goals should be individualized based on preexisting function and may include the ability to sit up or move in bed, move to a chair, work with physical therapy, or ambulate in the hallway. Protocols for the assessment for adverse effects are not well established. Because sedation typically precedes respiratory depression, it is generally recommended that patients are evaluated (eg, by nursing staff) for sedation after each opioid administration (10-20 minutes for intravenous and 30-60 minutes for oral administration based on the time-to-peak effect). Whether certain patients may benefit from more intensive respiratory monitoring, such as pulse oximetry or capnography, is an area of active investigation and not yet established.

\section{Prescribing at the Time of Hospital Discharge}

14. SHM recommends that clinicians ask patients about any existing opioid supply at home and account for any such supply when issuing an opioid prescription on discharge.

Even in the setting of acute pain, patients may have previously received an opioid prescription from an outpatient clinician prior to hospitalization. Unused prescription opioids create the possibility of both overdose (when patients take multiple opioids concurrently, intentionally or inadvertently) and diversion (many adults with prescription opioid misuse obtained their opioids from a friend or a relative who may or may not have known that this occurred ${ }^{47}$ ). The PDMP database can provide information related to the potential existence of any prior opioid supplies, which should be confirmed with the patient and considered when providing a new prescription on hospital discharge. Information on proper disposal should be provided if use of the preexisting opioid is no longer intended.
15. SHM recommends that clinicians prescribe the minimum quantity of opioids anticipated to be necessary based on the expected course and duration of pain that is severe enough to require opioid therapy after hospital discharge.

For many patients, the condition causing their acute pain will be mostly or completely resolved by the time of hospital discharge. When pain is still present at the time of discharge, most pain can be completely managed with nonopioid therapies. For those with ongoing pain that is severe enough to require opioids after hospital discharge, decisions regarding the duration of therapy should be made on a case-by-case basis; generally, however, provision of a 3- to 5-day supply will be sufficient, and provision of more than a 7-day supply of opioids should generally be avoided for several reasons. These include 1) acute pain lasting longer than 7 days after appropriate treatment of any existing underlying conditions should prompt re-evaluation of the working diagnosis and/ or reconsideration of the management approach, 2) receiving higher intensity opioid therapy (including longer courses) in the setting of acute pain has been associated with an increased risk of long-term disability and long-term opioid use, ${ }^{33,48,49}$ and 3) unused opioids create the possibility of intentional or unintentional opioid diversion (see Consensus Statement 14). ${ }^{47}$ Accordingly, clinicians should attempt to arrange an outpatient follow-up appointment for re-evaluation within 7 days, rather than providing an extended opioid prescription on hospital discharge. In situations where this is not feasible, and pain that is severe enough to require opioids is expected to persist longer than 7 days, an extended prescription may be indicated. However, some states have begun enacting legislation to limit the duration of first-time opioid prescriptions, typically using a 5-to-7 day supply as an upper limit; clinicians should be aware of and adhere to individual state laws governing their practice.

16. SHM recommends that clinicians ensure that patients and families or caregivers receive information regarding how to minimize the risks of opioid therapy for themselves, their families, and their communities. This includes but is not limited to 1) how to take their opioids correctly (the planned medications, doses, schedule); 2) that they should take the minimum quantity necessary to achieve tolerable levels of pain and meaningful functional improvement, reducing the dose and/or frequency as pain and function improve; 3) how to safeguard their supply and dispose of any unused supply; 4) that they should avoid agents that may potentiate the sedative effect of opioids, including sleeping medication and alcohol; 5) that they should avoid driving or operating heavy machinery while taking opioids; and 6) that they should seek help if they begin to experience any potential adverse effects, with inclusion of information on early warning signs. 
Clear and concise patient instructions on home opioid dosing and administration will limit opioid-related adverse events and dosing errors upon hospital discharge. Each of these recommendations derive from one or more of the existing guidelines and reflect the transfer of responsibility for safe opioid use practices that occurs as patients transition from a closely monitored inpatient setting to the more self-regulated home environment.

\section{DISCUSSION AND AREAS FOR FUTURE RESEARCH}

This Consensus Statement reflects a synthesis of the key recommendations from a systematic review of existing guidelines on acute pain management, adapted for a hospital-specific scope of practice. Despite a paucity of data on the comparative effectiveness of different management strategies for acute pain, several areas of expert consensus emerged across existing guidelines, which were felt to be relevant and applicable to the hospital setting. The objective of these recommendations is to provide information that can be used to inform and support opioid-related management decisions for acute pain by clinicians practicing medicine in the inpatient setting.

Although these recommendations are not intended to apply to the immediate perioperative setting (ie, care in the postanesthesia care unit), many of the recommendations in the existing guidelines upon which this Consensus Statement was based were intended for the postoperative setting, and, as others have noted, recommendations in this setting are mostly comparable to those for treating acute pain more generally. ${ }^{27}$ Those interested in pain management in the postoperative setting specifically may wish to review the recent guidelines released by the American Pain Society, ${ }^{50}$ the content of which is in close alignment with our Consensus Statement.

Several important issues were raised during the extensive external feedback process undertaken as part of the development of this Consensus Statement. Although many issues were incorporated into the recommendations, there were several suggestions for which we felt the evidence base was not sufficient to allow a clear or valid recommendation to be made. For example, several reviewers requested endorsement of specific patient education tools and opioid equivalency calculators. In the absence of tools specifically validated for this purpose, we felt that the evidence was insufficient to make specific recommendations. Validating such tools for use in the inpatient setting should be an area of future investigation. In the meantime, we note that there are several existing and widely available resources for both patient education (ie, opioid information sheets, including opioid risks, safe containment and disposal, and safe use practices) and opioid equivalency calculations that clinicians and hospitals can adapt for their purposes.

Several individuals suggested recommendations on communication with outpatient continuity providers around opioid management decisions during hospitalization and on discharge. Although we believe that it is of paramount importance for outpatient providers to be aware of and have input into these decisions, the optimal timing and the method for such communication are unclear and likely to be institution-specific depending on the availability and integration of electronic records across care settings. We recommend that clinicians use their judgment as to the best format and timing for assuring that outpatient physicians are aware of and have input into these important management decisions with downstream consequences.

Concerns were also raised about the time required to complete the recommended practices and the importance of emphasizing the need for a team-based approach in this realm. We agree wholeheartedly with this sentiment and believe that many of the recommended practices can and should be automated and/or shared across the care team. For example, PDMPs allow prescribers to appoint delegates to check the PDMP on their behalf. Additionally, we suggest that hospitals work to develop systems to assist care teams with performance of these tasks in a standardized and streamlined manner (eg, integrating access to the PDMP and opioid equivalency tables within the electronic health record and developing standard patient educational handouts). Provision of written materials on opioid risks, side effects, and safety practices may be helpful in facilitating consistent messaging and efficient workflow for members of the care team.

Finally, the working group carefully considered whether to include a recommendation regarding naloxone prescribing at the time of hospital discharge. The provision of naloxone kits to laypersons through Overdose Education and Naloxone Distribution Programs has been shown to reduce opioid overdose deaths ${ }^{51,52}$ and hospitalizations ${ }^{53,54}$ and is both safe and cost-effective. ${ }^{55}$ The Centers for Disease Control and Preventionrecommend that clinicians "consider offering naloxone to patients with a history of overdose, a current or past substance use disorder, receipt of $\geq 50 \mathrm{mg}$ of morphine equivalents per day or concurrent benzodiazepine use." ${ }^{11}$ However, these recommendations are intended for patients on chronic opioid therapy; presently, no clear evidence exists to guide decisions about the benefits and costs associated with prescribing naloxone in the setting of short-term opioid therapy for acute pain. Further research in this area is warranted.

The greatest limitation of this Consensus Statement is the lack of high-quality studies informing most of the recommendations in the guidelines upon which our Consensus Statement was based. The majority of recommendations in the existing guidelines were based on expert opinion alone. Additional research is necessary before evidence-based recommendations can be formulated.

Accordingly, the working group identified several key areas for future research, in addition to those noted above. First, ongoing efforts to develop and evaluate the effectiveness of nonopioid and nonpharmacologic management strategies for acute pain in hospitalized patients are necessary. Second, studies identifying the risk factors for opioid-related adverse events in hospitalized patients would help inform management decisions and allow deployment of resources and specialized monitoring strategies to patients at heightened risk. The working group also noted the need for research investi- 
gating the impact of PDMP use on management decisions and downstream outcomes among hospitalized patients. Finally, conversations around pain management and concerns related to aberrant behaviors are often challenging in the hospital setting owing to the brief, high-intensity nature of the care and the lack of a longstanding therapeutic alliance. There is a great need to develop strategies and language to facilitate these conversations.

In conclusion, until more high-quality evidence becomes available, clinicians can use the recommendations contained in this Consensus Statement along with their clinical judgment and consultation with pharmacists, interventional pain specialists, and other staff (eg, social work, nursing) to help facilitate consistent, high-quality care across providers and hospitals. We believe that doing so will help increase the appropriateness of opioid therapy, minimize adverse events, facilitate shared decision-making, and foster stronger therapeutic alliances at the outset of the hospitalization for patients suffering from acute pain.

\section{ACKNOWLEDGMENTS}

The authors would like to acknowledge and thank Kevin Vuernick, Jenna Goldstein, Meghan Mallouk, and Chris Frost, MD, from the SHM for their facilitation of this project and dedication to this purpose.

The authors would also like to thank the many individuals who provided comments on the draft recommendations, including the participants in the SHM RADEO program; the SHM members; the representatives of specialty societies, including the American Academy of Family Physicians, the American College of Physicians, the American Hospital Association, the American Society of Addiction Medicine, the American Society of Anesthesiologists, the American Society of Health-System Pharmacists, the Society of Critical Care Medicine, and the Society of General Internal Medicine; and the representatives of patient advocacy groups, including SHM PFAC, Regions Hospital Patient and Family Advisory Committee, Patient and Family Centered Care Council of St. Louis Children's Hospital, Missouri Family Partnership, and Parent and Family Care.

Disclosures: Dr. Herzig reports receiving compensation from the Society of Hospital Medicine for her editorial role at the Journal of Hospital Medicine (unrelated to the present work). Dr. Jena reports receiving consulting fees from Pfizer, Inc., Hill Rom Services, Inc., Bristol Myers Squibb, Novartis Pharmaceuticals, Vertex Pharmaceuticals, and Precision Health Economics, a consultancy to the life sciences industry (all unrelated to the present work). None of the other authors have any conflicts of interest to disclose.

Funding: The Society of Hospital Medicine (SHM) provided administrative assistance with the project and funded the in-person working group meeting but had no role in or influence on developing the content of the recommendations themselves. The SHM Board of Directors provided approval to submit the manuscript for publication without modification. Dr. Herzig was funded by grant number K23AG042459 from the National Institute on Aging. Dr. Mosher was supported in part by the Department of Veterans Affairs Office of Academic Affiliations and Office of Research and Development and Health Services Research and Development Service (HSR\&D) through the Comprehensive Access and Delivery Research and Evaluation Center (CIN 13-412). None of the funding agencies had involvement in any aspect of the study, including design, conduct, and reporting of the study.

\section{References}

1. Dowell D, Haegerich TM, Chou R. CDC Guideline for prescribing opioids for chronic pain-United States. JAMA. 2016;315(15):1624-1645.
2. United States Department of Veterans Affairs and Department of Defense. VA/DoD Clinical Practice Guideline for Opioid Therapy for Chronic Pain. https://www.healthquality.va.gov/guidelines/Pain/cot/. Accessed December 4, 2017.

3. Herzig SJ, Rothberg MB, Cheung M, Ngo LH, Marcantonio ER. Opioid utilization and opioid-related adverse events in nonsurgical patients in US hospitals. J Hosp Med. 2014;9(2):73-81.

4. Calcaterra SL, Yamashita TE, Min SJ, Keniston A, Frank JW, Binswanger IA. Opioid prescribing at hospital discharge contributes to chronic opioid use. $J$ Gen Intern Med. 2015;31(5):478-485.

5. Jena AB, Goldman D, Karaca-Mandic P. Hospital prescribing of opioids to medicare beneficiaries. JAMA Intern Med. 2016;176(7):990-997.

6. Mosher, HJ, B Hofmeyer, K Hadlandsmyth, KK Richardson, BC Lund. Predictors of long-term opioid use after opioid initiation at discharge from medical and surgical hospitalizations. J Hosp Med. 2018;13(4):XXX-XXX.

7. Barnett $M L$, Olenski $A R$, Jena $A B$. Opioid-prescribing patterns of emergency physicians and risk of long-term use. N Engl J Med. 2017;376(7):663-673.

8. Jones J, Hunter D. Consensus methods for medical and health services research. BMJ. 1995;311(7001):376-380.

9. Chang AK, Bijur PE, Esses D, Barnaby DP, Baer J. Effect of a single dose of oral opioid and nonopioid analgesics on acute extremity pain in the emergency department: a randomized clinical trial. JAMA. 2017;318(17): 1661-1667.

10. Moore RA, Derry S, Aldington D, Wiffen PJ. Single dose oral analgesics for acute postoperative pain in adults-an overview of Cochrane reviews. Cochrane Database Syst Rev. 2015;28(9):CD008659.

11. Moore RA, Derry S, Aldington D, Wiffen PJ. Adverse events associated with single dose oral analgesics for acute postoperative pain in adultsan overview of Cochrane reviews. Cochrane Database Syst Rev. 2015 Oct 13;(10):CD011407.

12. Holdgate A, Pollock T. Nonsteroidal anti-inflammatory drugs (NSAIDs) versus opioids for acute renal colic. Cochrane Database Syst Rev. 2005 Apr 18;(2):CD004137

13. Jones P, Dalziel SR, Lamdin R, Miles-Chan JL, Frampton C. Oral non-steroidal anti-inflammatory drugs versus other oral analgesic agents for acute soft tissue injury. Cochrane Database Syst Rev. 2015 Jul 1;(7):CD007789.

14. Smith $\mathrm{H}$, Bruckenthal P. Implications of opioid analgesia for medically complicated patients. Drugs Aging 2010;27(5):417-433.

15. Kessler ER, Shah M, S KG, Raju A. Cost and quality implications of opioid-based postsurgical pain control using administrative claims data from a large health system: opioid-related adverse events and their impact on clinical and economic outcomes. Pharmacotherapy 2013;33(4):383-391.

16. Minkowitz HS, Gruschkus SK, Shah M, Raju A. Adverse drug events among patients receiving postsurgical opioids in a large health system: risk factors and outcomes. Am J Health Syst Pharm. 2014;71(18):1556-1565.

17. Oderda G. Challenges in the management of acute postsurgical pain. Pharmacotherapy 2012;32(9):6S-11S.

18. Pawasauskas J, Stevens B, Youssef R, Kelley M. Predictors of naloxone use for respiratory depression and oversedation in hospitalized adults. Am J Health Syst Pharm. 2014;71(9):746-750.

19. Weingarten TN, Herasevich V, McGlinch MC, et al. Predictors of delayed postoperative respiratory depression assessed from naloxone administration. Anesth Analg 2015;121(2):422-429.

20. Calcaterra S, Glanz J, Binswanger IA. National trends in pharmaceutical opioid related overdose deaths compared to other substance related overdose deaths: 1999-2009. Drug Alcohol Depend. 2013;131(3):263-270.

21. Boscarino JA, Rukstalis M, Hoffman SN, et al. Risk factors for drug dependence among out-patients on opioid therapy in a large US health-care system. Addiction 2010;105(10):1776-1782.

22. Reid MC, Engles-Horton LL, Weber MB, Kerns RD, Rogers EL, O'Connor PG. Use of opioid medications for chronic noncancer pain syndromes in primary care. J Gen Intern Med. 2002;17(3):173-179.

23. Hall AJ, Logan JE, Toblin RL, et al. Patterns of abuse among unintentional pharmaceutical overdose fatalities. JAMA. 2008;300(22):2613-2620

24. Hasegawa K, Brown DF, Tsugawa Y, Camargo CA, Jr. Epidemiology of emergency department visits for opioid overdose: a population-based study. Mayo Clin Proc. 2014;89(4):462-471.

25. Washington State Agency Medical Directors' Group. Interagency guideline on prescribing opioids for pain. http://www.agencymeddirectors.wa.gov/ Files/2015AMDGOpioidGuideline.pdf. Accessed November 29, 2017.

26. Cantrill SV, Brown MD, Carlisle RJ, et al. Clinical policy: Critical issues in the prescribing of opioids for adult patients in the emergency department. Ann Emerg Med. 2012;60(4):499-525.

27. Hegmann KT, Weiss MS, Bowden K, et al. ACOEM practice guidelines: Opi- 
oids for treatment of acute, subacute, chronic, and postoperative pain. J Occup Environ Med. 2014;56(12):e143-e159.

28. Jena $A B$, Goldman D, Weaver $L$, Karaca-Mandic P. Opioid prescribing by multiple providers in Medicare: retrospective observational study of insurance claims. BMJ. 2014;348:91393.

29. Conrardy M, Lank P, Cameron KA, et al. Emergency department patient perspectives on the risk of addiction to prescription opioids. Pain Med. 2015;17(1):114-121.

30. Oderda GM, Said Q, Evans RS, et al. Opioid-related adverse drug events in surgical hospitalizations: impact on costs and length of stay. Ann Pharmacother. 2007;41(3):400-406.

31. Weingarten TN, Chong EY, Schroeder DR, Sprung J. Predictors and outcomes following naloxone administration during Phase I anesthesia recovery. J Anesth. 2016;30(1):116-122.

32. Miller M, Barber CW, Leatherman S, et al. Prescription opioid duration of action and the risk of unintentional overdose among patients receiving opioid therapy. JAMA Intern Med. 2015;175(4):608-615.

33. Deyo RA, Hallvik SE, Hildebran C, et al. Association between initial opioid prescribing patterns and subsequent long-term use among opioid-naive patients: a statewide retrospective cohort study. J Gen Intern Med. 2017:32(1):21-27.

34. Overdyk F, Dahan A, Roozekrans M, van der Schrier R, Aarts L, Niesters M. Opioid-induced respiratory depression in the acute care setting: a compendium of case reports. Pain Manag. 2014;4(4):317-325.

35. Wang Y, Sands LP, Vaurio L, Mullen EA, Leung JM. The effects of postoperative pain and its management on postoperative cognitive dysfunction. Am J Geriatr Psychiatry. 2007;15(1):50-59.

36. Daoust R, Paquet J, Lavigne G, Piette E, Chauny JM. Impact of age, sex and route of administration on adverse events after opioid treatment in the emergency department: a retrospective study. Pain Res Manag. 2015;20(1):23-28.

37. Al-Qadheeb NS, O'Connor HH, White AC, et al. Antipsychotic prescribing patterns, and the factors and outcomes associated with their use, among patients requiring prolonged mechanical ventilation in the long-term acute care hospital setting. Ann Pharmacother. 2013;47(2):181-188.

38. Compton WM, Volkow ND. Abuse of prescription drugs and the risk of addiction. Drug Alcohol Depend. 2006;83(1):S4-S7.

39. Bates DW, Cullen DJ, Laird N, et al. Incidence of adverse drug events and potential adverse drug events. Implications for prevention. ADE Prevention Study Group. JAMA. 1995;274(1):29-34.

40. Davies ED, Schneider F, Childs S, et al. A prevalence study of errors in opioid prescribing in a large teaching hospital. Int J Clin Pract. 2011;65(9):923-929.

41. Practice guidelines for acute pain management in the perioperative setting: an updated report by the American Society of Anesthesiologists Task Force on Acute Pain Management. Anesthesiology 2012;116:248-273.

42. Hah J, Mackey SC, Schmidt P, et al. Effect of perioperative gabapentin on postoperative pain resolution and opioid cessation in a mixed surgical cohort: a randomized clinical trial [published online ahead of print December 13, 2017]. JAMA Surg. doi: 10.1001/jamasurg.2017.4915

43. Garland EL, Baker AK, Larsen $\mathrm{P}$, et al. Randomized controlled trial of brief mindfulness training and hypnotic suggestion for acute pain relief in the hospital setting. J Gen Intern Med. 2017;32(10):1106-1113.

44. Wheeler M, Oderda GM, Ashburn MA, Lipman AG. Adverse events associated with postoperative opioid analgesia: a systematic review. J Pain. 2002;3(3):159-180.

45. Ramkumar D, Rao SS. Efficacy and safety of traditional medical therapies for chronic constipation: systematic review. Am J Gastroenterol. 2005;100(4): 936-971.

46. Abrahamsson T, Berge J, Ojehagen A, Hakansson A. Benzodiazepine, z-drug and pregabalin prescriptions and mortality among patients in opioid maintenance treatment-A nation-wide register-based open cohort study. Drug Alcohol Depend. 2017;174:58-64.

47. Han B, Compton WM, Blanco C, Crane E, Lee J, Jones CM. Prescription opioid use, misuse, and use disorders in U.S. adults: 2015 national survey on drug use and health. Ann Intern Med. 2017;167(5):293-301.

48. Franklin GM, Stover BD, Turner JA, Fulton-Kehoe D, Wickizer TM. Early opioid prescription and subsequent disability among workers with back injuries: the Disability Risk Identification Study Cohort. Spine 2008;33(2):199-204.

49. Webster BS, Verma SK, Gatchel RJ. Relationship between early opioid prescribing for acute occupational low back pain and disability duration, medical costs, subsequent surgery and late opioid use. Spine 2007;32(19):2127-2132.

50. Chou R, Gordon DB, de Leon-Casasola OA, et al. Management of postoperative pain: a clinical practice guideline from the American Pain Society, the American Society of Regional Anesthesia and Pain Medicine, and the American Society of Anesthesiologists' Committee on Regional Anesthesia, Executive Committee, and Administrative Council. J Pain. 2016;17(2):131-157.

51. McDonald R, Strang J. Are take-home naloxone programmes effective? Systematic review utilizing application of the Bradford Hill criteria. Addiction 2016;111(7):1177-1187

52. Mueller SR, Walley AY, Calcaterra SL, Glanz JM, Binswanger IA. A review of opioid overdose prevention and naloxone prescribing: implications for translating community programming into clinical practice. Substance abuse 2015;36(2):240-253.

53. Walley AY, Xuan Z, Hackman $\mathrm{HH}$, et al. Opioid overdose rates and implementation of overdose education and nasal naloxone distribution in Massachusetts: interrupted time series analysis. BMJ. 2013;346:f174.

54. Wheeler E, Jones TS, Gilbert MK, Davidson PJ. Opioid overdose prevention programs providing naloxone to laypersons-United States, 2014. MMWR. 2015;64(23):631-635.

55. Coffin PO, Sullivan SD. COst-effectiveness of distributing naloxone to heroin users for lay overdose reversal. Ann Intern Med. 2013;158(1):1-9. 